\title{
Quadrupedal locomotion based on a muscular activation pattern with stretch-reflex
}

\author{
Andre Rosendo, Shogo Nakatsu, Xiangxiao Liu, Masahiro Shimizu and Koh Hosoda
}

\begin{abstract}
Cyclical locomotion, such as walking, hopping and running, is known to be generated at the spinal cord, guiding human and animal strides over different gaits. Over the last years, many researchers concentrated their study on the origin of such signals, replicating them by either controlling joint angles or torques. In this work, we use a quadruped pneumatic robot to reproduce stable walking on a treadmill through a muscular activation pattern. Unlike previous studies, neither angles or torques are taken into consideration. Similarly to biological morphology, with variating moment arms, muscles contract rhythmically and their inherent compliance adapts to the floor. Proportional feedback upon touching the floor (stretch-reflex) is also tested, and its effects are explained. In the future, this methodology can be used to produce adaptive gait and improve current robotic by exploring interaction between control and soft bodies.
\end{abstract}

\section{INTRODUCTION}

The gait stability observed in biped and quadruped animals has been the main inspiration for robotics in the recent time. From humans transitioning from walking to running [1], to frogs jumping 50 body lengths and landing properly [2], natural locomotion intrigues and inspires scientists.

For over one hundred years [3], biologists have been trying to decipher the code which generates such locomotion. Beyond analyzing electromyographic patterns and joint angles during stable walking [4], [5], [6], [7], recent biological research approaches locomotion by splitting it in parts and analyzing each one individually. Animals control their bodies through a combination of higher and lower control signals; the first is controlled by the brain that reads multiple sensors, like vision and vestibular signals to plan paths, while the latter is controlled by the spinal cord that provides low-level feedforward and feedback[8]. In this vein, Pearson et al. [9], [10] performs a series of experiments with decerebrate cats, isolates brain signals and analyzes muscular stretch-reflex finishing stance phase. Gorassini et al. [11] conducts an ingenious experiment where a trap door opens milliseconds before a cat steps into it, recording the corrective response of the cat. With response times as low as 30 milliseconds, they prove that low-level muscular signals are responsible for such adaptive gait, where a brain-signal would take more than 100 milliseconds to react to such stimulus.

Seeking to improve current robotic behavior, a few roboticists have started applying such segmented control method in bio-inspired robots. Initial research on bio-inspired control

This work was aided by KAKENHI Kiban(S) 23220004 and 25540117. The authors are with Multimedia Department, Faculty of Information Science and Technology, Osaka University, 1-5 Yamadaoka, Suita, Osaka, Japan. andre.rosendo@ist.osaka-u.ac.jp focused on simple animals, such as lampreys and salamanders [12], and later expanded to quadruped animals [13]. A quadruped cheetah-inspired robot moves accordingly to central pattern generated (CPG) signals, with compliant elements in its legs and being actuated by electric motors [14]. One step closer to biology, Tsujita et al. [15] show a statically stable simplified robot, with only two links per leg, achieve gait transitions by coordinating its muscles. From another perspective, in [16], [17] a biomimetic robot, adopting cheetah-inspired links, air muscles and moment arms, walks on the treadmill with a control method solely based on stretch-reflex. Geyer and Herr [18] simulated in a computer environment a walking humanoid using stretchreflex to coordinate its steps.

Although CPG research is well known, the use of such architecture to activate muscles or its combination with stretchreflexes is yet to be fully investigated. In this work, we use a quadruped pneumatic robot to reproduce a stable walking. Instead of extracting data from biological experiments, we opted to build a biomimetic robot with dimensions, link lengths and moment arms inspired on biological data [19], [20], [21], adopting a constructivist approach. The robot walks on a treadmill using a Muscular Activation Pattern (MAP), which does not take into account angles or torques, and adds an incremental muscle contraction to its distal muscles' rhythmic pattern. We hypothesize that such incremental contraction should be proportional to the ground reaction force (GRF) to simulate stretch-reflex, as observed in research with running birds [22].

In the future, more experiments will be conducted, exploring other stimuli which might create an incremental contraction (joint deformation, artificial muscle deformation per se, etc.) and their role on stability. Adaptive legged locomotion is present in the simplest of the lifeforms [23], and a novel perspective on robotics might close the gap between robots and animals.

\section{MATERIALS AND METHODS}

Here, a biomimetic robot was used to reproduce phenomena observed in real animals. Initially, the design considerations and behavior of the adopted air muscles will be described, followed by a detailed explanation of the proposed MAP and its differences from usual CPGs.

\section{A. Robot Design}

The robot design considers, initially, overall dimensions from its biological counterpart (height, width, length). Forelimbs, hindlimbs and inter-girdle measurements were taken 


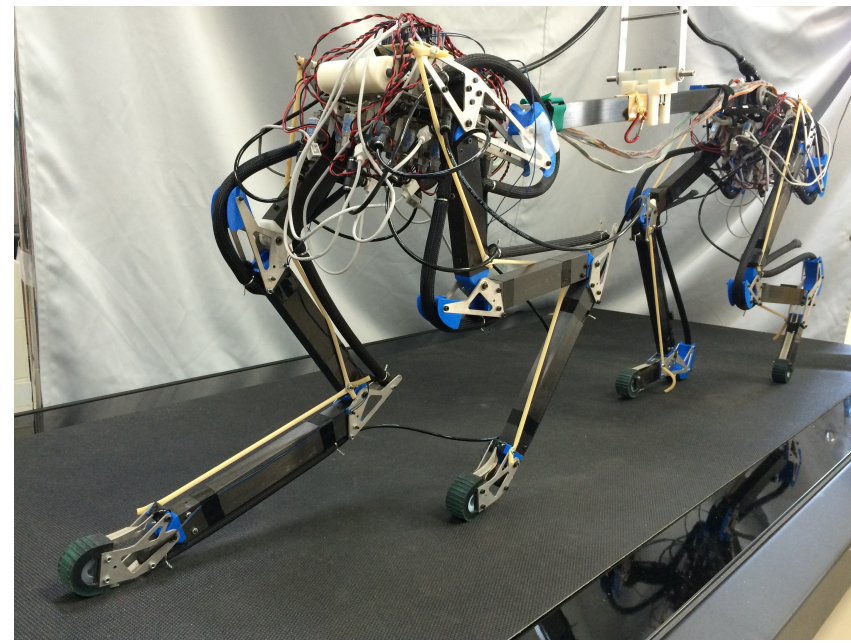

Fig. 1. Full body robot with 14 degrees of freedom. Aiming to concentrate on the low-level control methodology 14 air muscles control its joints, evaluating spinal and muscular contributions.

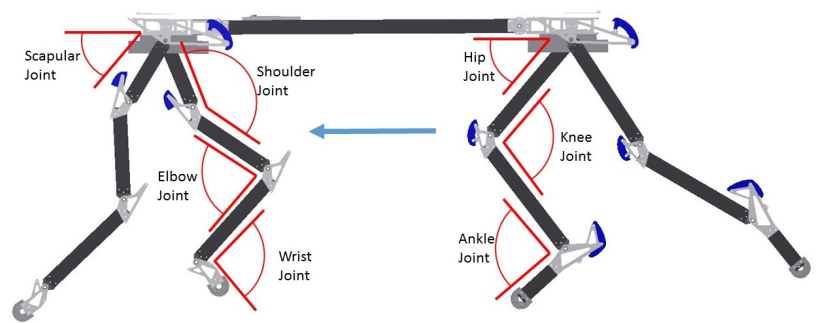

Fig. 2. Pictograph with all seven ipsilateral joints of the robot. The design choice of implementing a scapula was made due to the extra range of motion provided by this joint. The wrist joint, on the other hand, provides extra clearance during swing phase. For the sake of simplicity all joints flex when their angles decrease, extending otherwise.

from published data (cheetah's dissection [19] [20], markerbased observation [21], and video observation) and considered to create a realistic representation of the animal. A picture of the final robot can be found in Fig. 1.

In this work, aiming to focus on low-level control methodologies, the previously adopted redundant structure was highly simplified, resulting in a robot with the same number of muscles as the number of joints. This robot possesses 14 degrees of freedom (DOF) and 14 muscles, and a pictograph illustrating each degree of freedom can be seen in Fig. 2. A flexible spine is a very important design feature, but aiming to concentrate on low-level controllability the complexity of the system was reduced, and thus not implemented. A fourth proximal joint on the forelimbs, however, was added, since locomotion would be severely hindered without this extra DOF [20].

The tip of the forelimb is formed by a wrist joint, which similarly to animal locomotion avoids collision with the floor during swing phase. The angles represented in Fig. 2 decrease with flexion, increasing with extending movements. Each joint possesses a moment arm which was designed to resemble the one present in real animals. As noted by [19], small moment arms prioritize fast movements while large

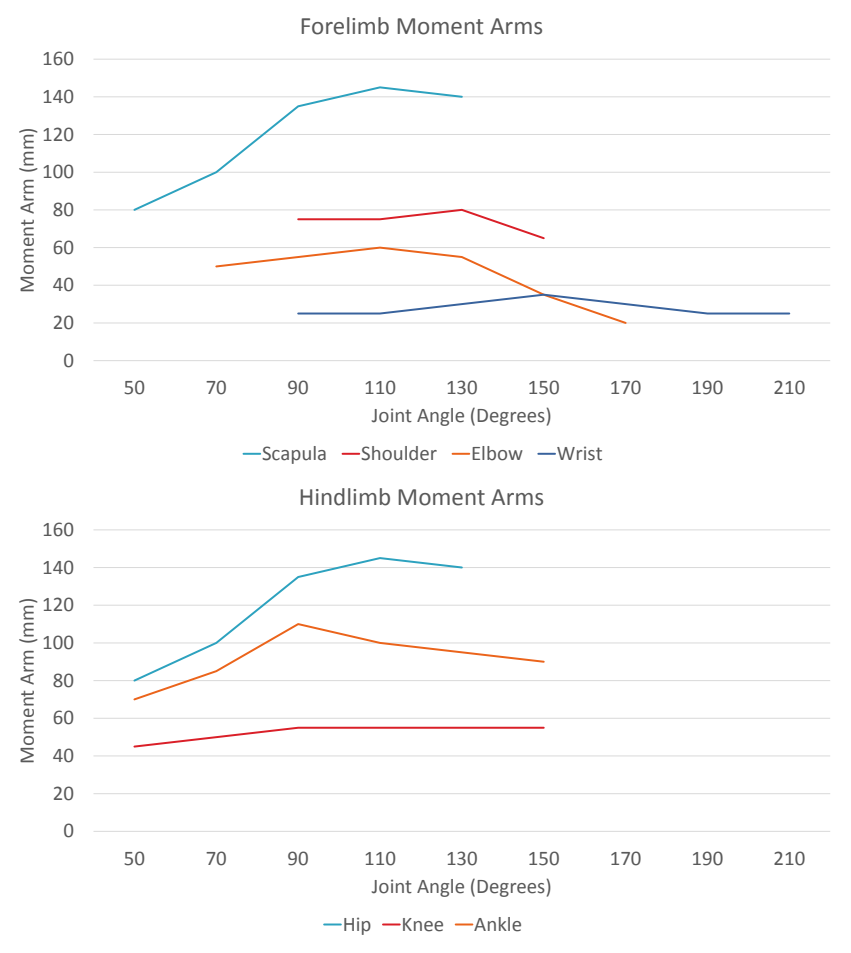

Fig. 3. Graph with continuously changing moment arms. Forelimbs and hindlimbs share the same design construction for the most proximal joint, hence the same behavior between scapular and hip joints. Knees and wrists possess an almost constant moment arm, with very small values, while the other joints present higher values and complex behavior.

moment arms better translate muscle force into torque. In Fig. 3, the length of the moment arms for each joint is plotted. Muscular torque changes during gait, with distal joints usually decreasing their moment arms as full extension is reached (e.g. elbow) and proximal joints increasing their torque with extension (maximum torque at the end of stance phase).

The muscle choice was made considering function and power output. After analyzing data from dissected cheetah's limbs, our muscle choices were:

- Flexor carpi ulnaris/radialis (FC) - Strong wrist flexor, being activated during swing phase. Monoarticular and only acting over one joint.

- Triceps brachi (TB) - As in human anatomy, in the anatomy of the cheetah this muscle is a powerful elbow extensor and acts over two joints (biarticular). Has the scapula and the ulna/radius as attachment points.

- Supraspinatus (SS) - One of the five biggest muscles on the cheetah's forelimbs, this muscle is monoarticular and extends the shoulder joint.

- Serratus ventralis (SV) - This muscle is responsible for the extension of the scapular joint. It is hypothesized in [20] that this monoarticular muscle is important for fast locomotion, where it increases the range of motion of the animal.

- Gastrocnemius (GA) - Powerful biarticular muscle composed of $61 \%$ of fast-twitching fibers [24], this muscle 
extends the ankle joint. It was chosen over soleus due to its bigger size and biarticular properties.

- Vastus lateralis (VA) - Monoarticular muscle responsible for extending the knee joint, composed of $83 \%$ of fasttwitching fibers [24].

- Biceps femoris (BF) - Strong monoarticular muscle that acts over the hip joint to extend the hip joint.

Rubberactuators (air muscles) were chosen as power source due to their great deformability (highly needed in adaptive behavior), high force output and low weight. These air muscles have a non-linear behavior (similarly to biological muscles) and are supplied with compressed air through pneumatic valves, which are connected to a microcontroller.

\section{B. Control}

The controller is divided in two sections: feed-forward (spine signals) and feedback (muscle signals). In the feedforward control, four limbs are coupled and phase differences are enforced between fore-hind limbs and also right-left sides. Sinusoidal patterns are created, similarly to traditional CPG methods [12], and used to control the pressure inside each muscle. In Fig. 4, we can see that the sinusoidal pattern is broken regularly with sudden peaks, and the maximum reference level is not reached, being limited by the maximum muscle pressure of $0.65 \mathrm{MPa}$.

The existence of ripples on the pressure values is due to the control choice, which is based on on-off valves instead of proportional valves. The better behavior of proportional valves is hindered by its inability to supply air in high flow rates, being inadequate for fast contractions. A P control is used to enforce the tracking of the sinusoidal reference value and Hysteresis control method is used to command the valves, being responsible for the small delay between reference and actual pressures.

The differences between activating compliant muscles and enforcing torque/angle on joints emerges when contact with the floor happens. Even without considering feedback signals (feedback contribution will be discussed below), joint angle control takes little consideration to ground differences while torque control does not consider torque differences from changing moment arms or muscular elastic force.

The proposed feedback control continuously samples the force sensor at the tip of each limb, producing a proportional pressure increment whenever the signals pass a preset threshold, which amplifies the force against the floor. The pressure increment $\Delta_{P}$ can be defined as:

$$
\Delta_{P}= \begin{cases}F_{\text {sens }} x, & \text { if } F_{\text {sens }} \geq 1.8 \mathrm{~V} \\ 0, & \text { otherwise }\end{cases}
$$

where $F_{\text {sens }}$ is the voltage output from the force sensor and $x$ is a converting constant $(\mathrm{MPa} / \mathrm{V})$, translating the input into pressure $(\mathrm{MPa})$. The threshold of $1.8 \mathrm{~V}$ was chosen for being the value which every paw was surely in stance phase.

Before experiments, the proposed feedback methodology was used to produce incremental pressure on walking hindlimbs, as it can be seen in Fig. 5. There, an incremental

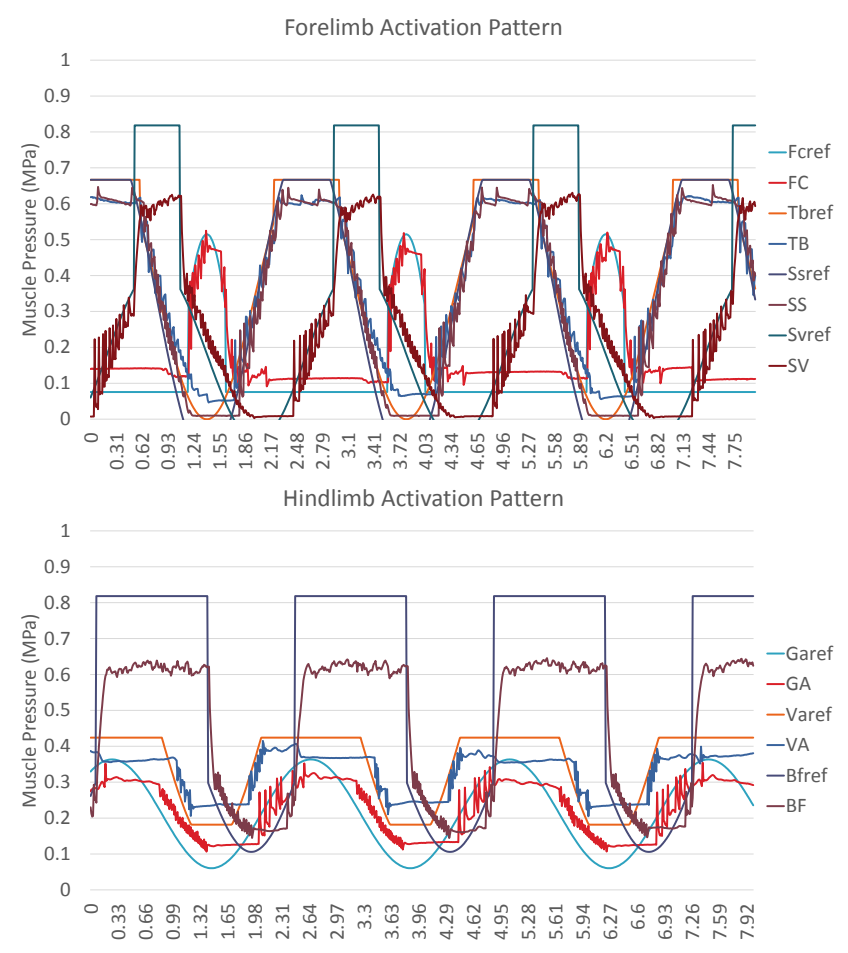

Fig. 4. Graph with Muscular Activation Pattern without feedback. Forelimbs and hindlimbs alternate between flexion and extension by contracting and relaxing their muscles. Ripples present on pressure signals are an effect of the adopted on-off valves. The maximum pressure is limited at $0.65 \mathrm{MPa}$

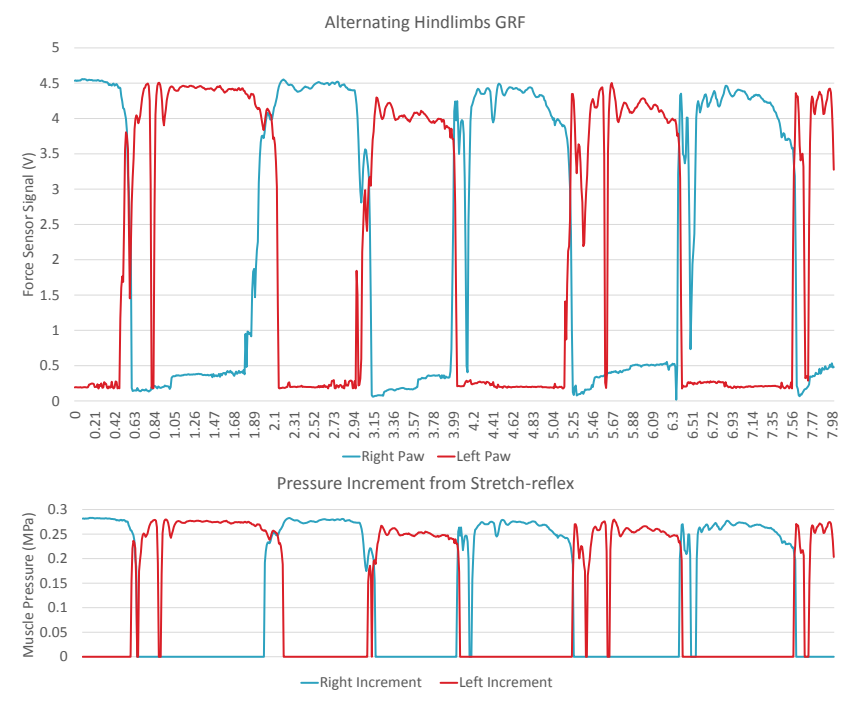

Fig. 5. Graph with alternating hindlimbs, registering their contact against the floor. The pressure increment shown at the bottom graph is proportional to signals from the force sensor, representing an stretch-reflex effect.

pressure of $0.28 \mathrm{MPa}$ is being generated, which adds to the cyclical pattern from spinal signals.

\section{EXPERIMENTS}

The robot was attached to a slider and its walking gait was constrained in two dimensions while the feed-forward method was used to reproduce a quadruped walk on a 


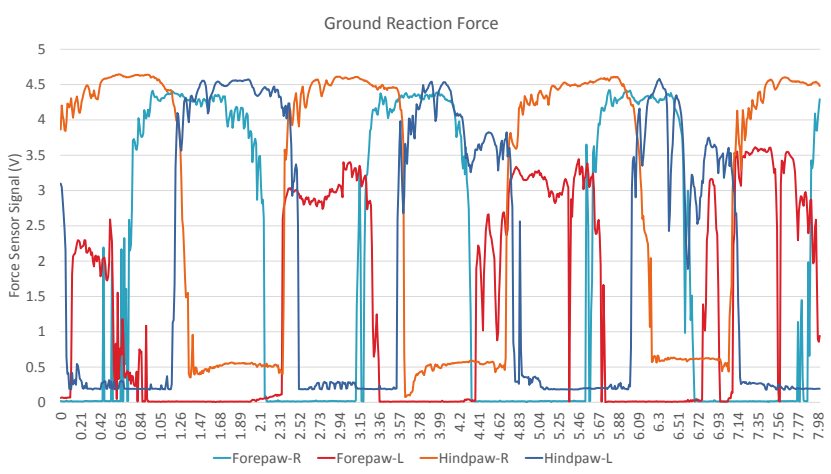

Fig. 6. Graph with force sensor output from four paws touching the floor, alternatively, while using feed-forward control. Right and left values alternate between themselves, keeping gait stability. Value peaks change between sensors, which are curved to cover the entire paw, affecting repeatability.

treadmill. The programming had to be tuned to reproduce a stable walking, with maximum and minimum pressure values manually adjusted.

\section{A. From spinal cord rhythmic signals to muscles}

After series of trials, a walk on the treadmill was successfuly achieved solely using feed-forward signals (average of 40 steps per treadmill walk). Feed-forward signals being used as a control method is a common research approach, with dozens of papers exploring this issue and an evergrowing complexity of its rhythmic patterns. The novelty of this experiment is not based on the creation of complex patterns to produce adaptive gaits, but to prove that simple patterns can also generate stability if used to control soft muscles, directly.

The biological idea of position and force is directly related to proprioception, where muscular deformation is sensed and an estimative of angle and torque is calculated in our brains. Controlling muscular contraction is more intuitive, where constant muscle pressure does not constrain a joint to a specific angle (different angles can be reached due to muscular elasticity) nor to a specific torque (different output forces can be reached, depending on the joint angle and moment arms).

During feed-forward-based control force sensors were sampled, and in Fig. 6 we can see the collected data from four different paws during walking on a treadmill. Signals between right and left sides are alternated, and a small signal asymmetry can be seen between their peaks (specially on forelimbs). This asymmetry is attributed to the low repeatability of the adopted force sensing resistors, and future works will adopt different sensing approaches.

Solely using feed-forward control, stability is kept for ten steps by each leg on the average, with the robot either falling forward (forelimbs do not land sufficiently ahead of the robot) or back (hindlimbs land too much apart from each other, similarly to humans taking a very long stride, which destabilizes the system when the back leg is removed). The consideration of muscle signals might help overcome this

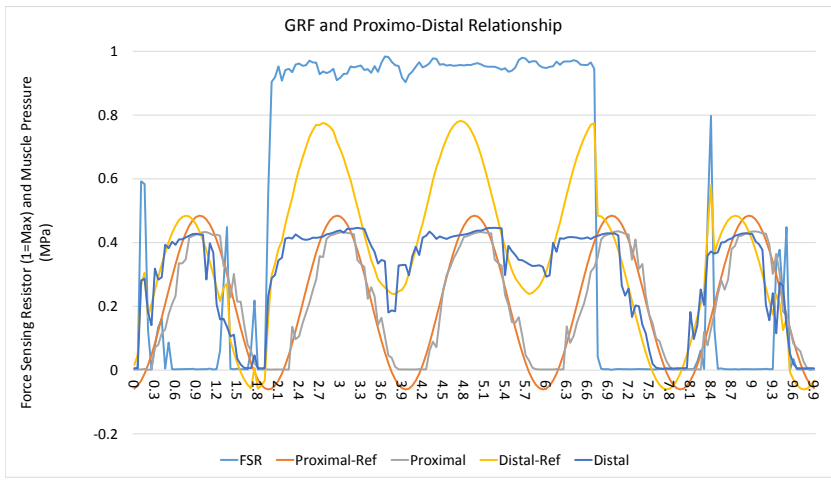

Fig. 7. Graph with force sensor output from one paw while using feed-forward control with feedback muscle increment. Proximal and distal muscles are also depicted. In this experiment the robot air walks while the paws are randomly excited.

problem, where 1. the decrease of GRF at the end of stance phase can be used as a trigger to a faster stance to swing transition [17] and 2. the increase of the same force could be used to "stiffen" the leg, similarly to a stretch-reflex.

\section{B. Stretch-reflex effect on gait}

Seeking to understand stretch-reflex effect, we designed an experiment to register the effect of muscle pressure increment (stretch-reflex) on the rhythmic MAP. The robot was lifted from the treadmill and forced to perform an "air walk" without floor contact. Then, the foot tip of the robot is manually excited and the effects of the generated muscle increment on the original MAP are studied (see Fig. 7).

Reference and actual pressure values for distal and proximal muscles show that while proximal values are unaffected by floor feedback, distal members react proportionally to the force applied upon them. This method, inspired by research with running birds [22], has two positive outcomes on gait:

1) During swing phase, the consequences of hitting an obstacle are drastic for any legged system. In Fig. 7 , even during swing phase the muscular increment is valid, helping the robot overcome obstacles by contracting the muscles, creating an additional stance phase (temporary double support).

2) During touchdown, the initial impact with the floor creates a distal contraction, which helps establish the new stance phase. This is a very helpful feature, since body inclination (pitch) continuously changes during walking, and the leg touchdown angle should also adapt to these changes.

As explained in [22], the proximal joints are not affected by gait disturbances, keeping its cyclical behavior unchanged. Snapshots of the robot walking on a treadmill is depicted in Fig. 8, and a video with the robot walking on a treadmill is also available ${ }^{1}$.

\section{CONCLUSIONS AND FUTURE WORKS}

We used a quadruped pneumatic robot to reproduce a stable walking on a treadmill while following a rhythmic

\footnotetext{
${ }^{1}$ Video available at www.robo-sapiens.com/Research
} 


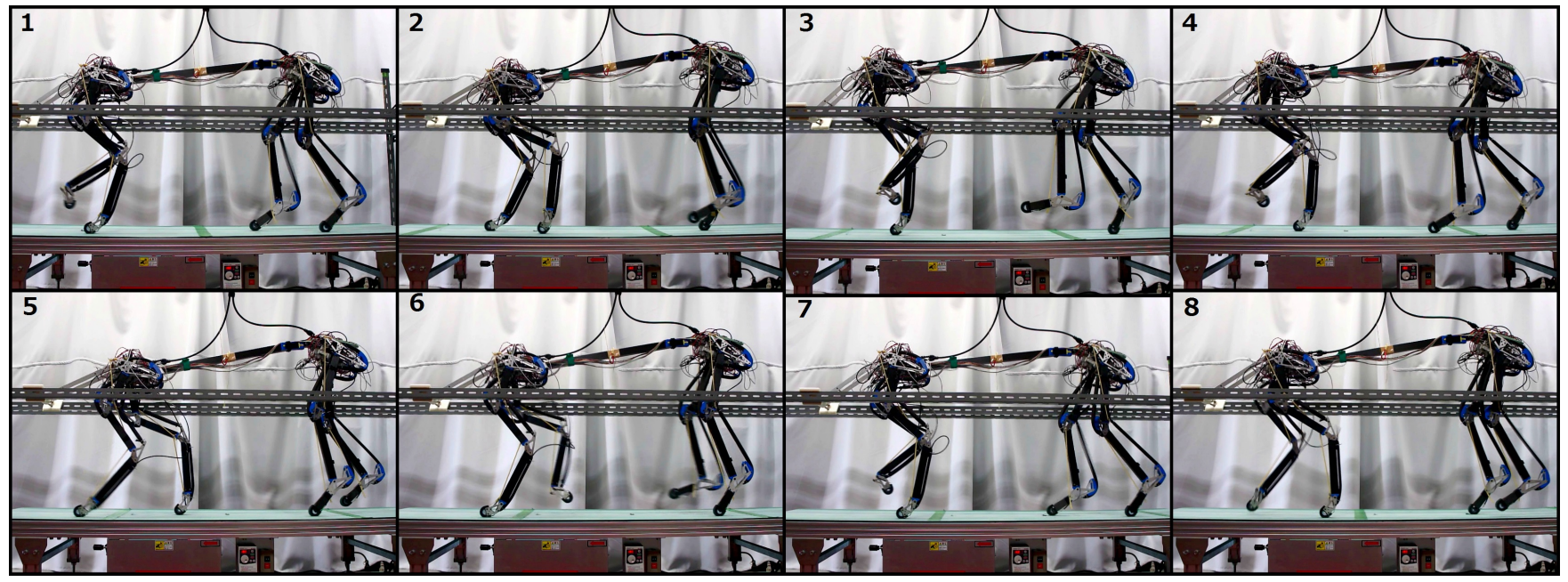

Fig. 8. Snapshots of Pneupard walking on the treadmill. In the top 4 snapshots we can see left hindlimb entering swing phase (2), forcing the left forelimb to do the same (3) when finishing swing to enter stance phase. The same pattern observed on the left side is repeated on the right through snaps (5-8).

pattern which actuates muscles directly (MAP). Differently from previous researches on feed-forward patterns, neither angles or torques are taken in consideration. Similarly to biological morphology, with variating moment arms, muscles contract rhythmically and their inherent compliance adapts to the floor. In a first experiment, the quadruped robot adopted the feed-forward control and successfully walked on a treadmill. In a second experiment, the effects of a stretch-reflex control on muscles were evaluated outside the treadmill.

While in [15] air muscles are controlled by a CPG architecture, its body morphology is statically stable and makes locomotion easier without falling. Contribution from stretch-reflex are not considered and feedback is solely used as a parameter to change joint frequency instead of muscle contraction.

Stretch-reflex is an extremely adaptive phenomenon observed in animals, and a combination of this feature with feed-forward methods can improve stability over disturbances. Feed-forward control, on the other hand, can be used with different gradients; a higher influence on proximal joints enforces coordination while lower influences on distal links encourages adaptativity from feedback signals. In the future, this methodology will be used to produce adaptive gait and improve current robotic by exploring interaction between control and soft bodies.

\section{A. Future Works}

The contribution from stretch-reflex during actual walking was not evaluated and it will be our next stepping stone to reach higher stability during walking. Another important future work is the integration of muscular unloading rule (triggering transition from stance to swing) to our proposed MAP.

\section{REFERENCES}

[1] V. Segers, P. Aerts, M. Lenoir and D. De Clerq, Dynamics of the body centre of mass during actual acceleration across transition speed, $J$. Exp. Biol., vol. 210, 2007, pp 578-585.
[2] R.S. James and R.S. Wilson, Explosive Jumping: Extreme morphological and physiological specializations of Australian rocket frogs (Litoria nasuta, Physiol. and Biochem. Zool., vol. 81, 2008, pp 176-185.

[3] E. Muybridge, Animals in Motion, Chapman and Hall, Ltd., London; 1899.

[4] I. Engberg and A. Lundberg, An electromyographic analysis of muscular activity in the hindlimb of the cat during unrestrained locomotion, Acta Physiol. Scand., vol. 75, 1969, pp. 614-630.

[5] G.E. Goslow Jr., R.M. Reinking, and D.G. Stuart, The cat step cycle: hind limb joint angles and muscle lengths during unrestrained locomotion, J. Morphol., vol. 141, 1973, pp. 1-42.

[6] A. English, An electromyographic analysis of forelimb muscles during overground stepping in the cat, J. Exp. Biol., vol. 76, 1978, pp. 105122.

[7] B.I. Prilutsky, W. Herzog, and T.L. Allinger, Mechanical power and work of cat soleus, gastrocnemius and plantaris muscle during locomotion: possible functional significance of muscle design and force patterns, J. Exp. Biol., vol. 199, 1996, pp. 801-814.

[8] C. Paul, M. Belloti, S. Jezernik and A. Curt, Development of a human neuro-musculo-skeletal model for investigation of spinal cord injury, Biol. Cybern, vol. 93, 2005, pp. 153-170.

[9] T. Lam and K.G. Pearson, The role of proprioceptive feedback in the regulation and adaptation of locomotor activity, in Sensorimotor Control of Movement and Posture, Kluwer Academic, New York, NY; 2002.

[10] G.W. Hiebert and K.G. Pearson, Contribution of sensory feedback to the generation of extensor activity during walking in the decerebrate cat, J Neurophysiol., vol. 81, 1999, pp. 758-770.

[11] M.A. Gorassini, A. Prochazka, G.W. Hiebert and M.J.A. Gauthier, Corrective response to loss of ground support during walking. 1. Intact cats, J. Neurophysiol., vol. 71, 1994, pp. 603-610.

[12] A.J. Ijspeert, Central pattern generators for locomotion in animals and robots: a review, Neural Networks, vol. 21, 2008, pp. 642-653.

[13] A.J. Ijspeert, A. Crespi, D. Ryczko, and J.-M. Cabelguen, From swimming to walking with a salamander robot driven by a spinal cord model, Science, vol. 315, 2007, pp. 14161420.

[14] S. Rutishauser, A. Sproewitz, L. Righetti and A.J. Ijspeert, "Passive compliant quadruped robot using central pattern generators for locomotion control", in Proc. IEEE Intl. Conf. on Biomedical Robotics and Biomechatronics, 2008.

[15] K. Tsujita, T. Kobayashi, T. Inoura and T. Masuda, "Gait transition by tuning muscle tones using pneumatic actuators in quadruped locomotion", in Proc. Intl. Conf. on Intelligent Robots and Systems, 2008, pp. 2453-2458.

[16] A. Rosendo, S. Nakatsu, K. Narioka and K. Hosoda, "Pneupard: A biomimetic musculoskeletal approach for a feline-inspired quadruped robot", in Proc. IEEE Intl. Conf. on Intelligent Robots and Systems, 2013.

[17] A. Rosendo, S. Nakatsu, K. Narioka and K. Hosoda, Producing 
alternating gait on uncoupled feline hindlimbs: muscular unloading rule on a biomimetic robot, Advanced Robotics, vol. 28, 2014, pp. 351-365.

[18] H. Geyer and H. Herr, A muscle-reflex model that encodes principles of legged mechanics produces human walking dynamics and muscle activities, IEEE Trans. Neural Sys. and Rehab. Eng., vol. 18, 2010, pp. 263-273.

[19] P.E. Hudson, A.M. Wilson et al., Functional anatomy of the cheetah (Acinonyx Jubatus) hindlimb, J. Anat., vol. 218, 2011, pp. 363-374.

[20] P.E. Hudson, A.M. Wilson et al., Functional anatomy of the cheetah (Acinonyx Jubatus) forelimb, J. Anat., vol. 218, 2011, pp. 375-385.

[21] L. Day and B. Jayne, Interspecific scaling of the morphology and posture of the limbs during the locomotion of cats (Felidae), J. Exp. Biol., vol. 210, 2007, pp. 642-657.

[22] M.A. Daley, G. Felix and A.A. Biewener, Running stability is enhanced by a proximo-distal joint neuromechanical control, J. Exp. Biol., vol. 210, 2007, pp. 383-394.

[23] R. Kukillaya, J. Proctor and P. Holmes, Neuromechanical models for insect locomotion: Stability, maneuverability, and proprioceptive feedback, Chaos, vol. 19, 2009, 026107.

[24] T.M. Williams, G.P. Dobson, O. Mathieu-Costello, D. Morsbach, M.B. Worley and J.A. Phillips, Skeletal muscle histology and biochemestry of an elite sprinter, the African cheetah, J. Comp. Physiol. B, vol. 167, 1997, pp. 527-535. 